\title{
High Power Supercontinuum Generation in Graded-Index Multimode Fibers
}

\author{
Uğur Teğin ${ }^{1,2}$ and Bülend Ortaç ${ }^{1}$ \\ 1. National Nanotechnology Research Center and Institute of Materials Science and Nanotechnology, Bilkent University, 06800 Ankara, \\ Turkey \\ 2. Present address: Optics Laboratory and Laboratory of Applied Photonics Devices, Ecole Polytechnique Federale de Lausanne, Lausanne,
} Switzerland

Over the years, supercontinuum generation in fibers are studied extensively. Photonic crystal fiber technology detailed these studies by allowing the change of dispersion parameter. Nowadays, multimode fibers attracted huge attention by enabling spatiotemporal nonlinearities and multimodal interactions. Recently, with graded-index multimode fibers, researchers reported new nonlinear dynamics such as cascaded Raman scattering [1], spatiotemporal instability [2,3], self-beam cleaning [4], multimode solitons [5].

In this letter, we report cascaded Raman scattering based novel method to generate octave-spanning high power and high repetition rate supercontinua in graded-index multimode fibers. We develop an all-fiber laser to obtain pump pulses with $\mathrm{MHz}$ repetition rates, $\sim 30 \mathrm{~kW}$ peak power and 70 ps pulse duration at $1040 \mathrm{~nm}$ to excite the graded-index MMFs. We tested $10 \mathrm{~m}$ and $20 \mathrm{~m}$ fiber length to investigate the supercontinuum generation (Fig.1.a). Output beam profiles is investigated for $730 \mathrm{~nm}$ to $1200 \mathrm{~nm}$ and $1100 \mathrm{~nm}$ to $1200 \mathrm{~nm}$ range and both cases feature Gaussian distribution with higher order background (Fig.1.b-c). When we investigate the formation of supercontinuum we observed that cascaded Raman scattering is the driven nonlinear force on frequency conversion (Fig.1.d-e). From $200 \mathrm{kHz}$ to $2 \mathrm{MHz}$ repetition rate, we scale our pump power by remaining peak power fixed and demonstrated the power scaling capability of multimode fibers.
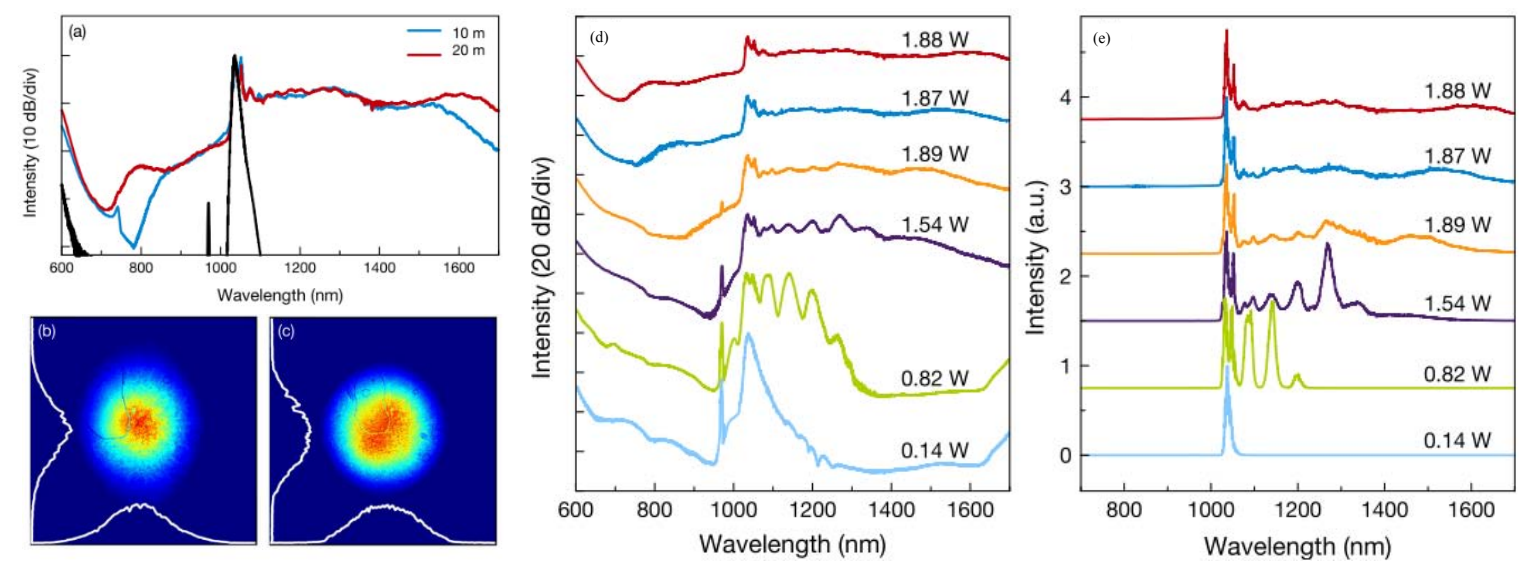

Fig. 1 (a) Supercontinuum generation for $10 \mathrm{~m}$ and $20 \mathrm{~m}$ fiber lengths. (b) and (c), Measured output beam profile $730 \mathrm{~nm}$ to $1200 \mathrm{~nm}$ and $1100 \mathrm{~nm}$ to $1200 \mathrm{~nm}$ range, respectively. (d) and (e) Formation of supercontinuum presented in details with logarithmic and linear scaling.

Experimental and numerical studies reveal that unique cascaded stimulated Raman scattering observed in graded-index MMF plays a significant role in the octave-spanning spectral evolution for the first time. The highest supercontinuum output power of $3.96 \mathrm{~W}$ is achieved in graded-index multimode fiber with $62.5 \mu \mathrm{m}$ core diameter. We also investigated the effect of fiber core to supercontinuum generation by comparing fiber with $50 \mu \mathrm{m}$ core diameter with aforementioned results. We observe similar wavelength conversion mechanism with $50 \mu \mathrm{m}$ core diameter fiber as well. The presented novel method demonstrates a way to generate high power and high repetition rate supercontinuum sources for application purposes in different research fields such as microscopy and imaging.

\section{References}

[1] H. Pourbeyram, G. Agrawal, and A. Mafi, "Stimulated Raman scattering cascade spanning the wavelength range of 523 to $1750 \mathrm{~nm}$ using a graded-index multimode optical fiber," Appl. Phys. Lett. 102, 201107 (2013).

[2] K. Krupa, A. Tonello, A. Barthélémy, V. Couderc, B. M. Shalaby, A. Bendahmane, S. Wabnitz, "Observation of geometric parametric instability induced by the periodic spatial self-imaging of multimode waves,” Phys. Rev. Lett., 116, 183901. (2016).

[3] U. Teğin, and B. Ortaç, "Spatiotemporal Instability of Femtosecond Pulses in Graded-Index Multimode Fibers" IEEE Photonics Technology Letters 29, 2195 (2017).

[4] K. Krupa, A. Tonello, B. M. Shalaby, M. Fabert, A. Barthélémy, G. Millot, V. Couderc, "Spatial beam self-cleaning in multimode fibres". Nat. Photonics, 11, 237 (2017).

[5] W. H. Renninger, F. W. Wise, “Optical solitons in graded-index multimode fibres”. Nat. Commun, 4, 1719 (2013). 